\title{
Different presentation of pulmonary parenchymal disruption in COVID-19 pneumonia. Case series of Sub-Intensive Care Unit in Naples, Italy
}

\author{
Giorgio E. Polistina, Francesca Simioli, Pasquale Imitazione, Maurizia Lanza, Anna Annunziata, \\ Giuseppe Fiorentino
}

Sub-Intensive Care Unit and Respiratory Physiopathology Department, Cotugno-Monaldi Hospital, Naples, Italy

\section{Established facts}

- Chest high resolution computed tomography (HRCT) is an essential tool in diagnosis and management of COVID-19 pneumonia

- Pulmonary parenchymal opacity is a typical sign of the disease

- The most common patterns and distribution of CT findings in COVID-19 pneumonia are ground glass opacities (88.0\%), bilateral involvement (87.5\%), peripheral distribution (76.0\%), and multi lobar (more than one lobe) involvement $(78.8 \%)$

\section{Novel insights}

- Pneumothorax, pneumomediastinum, bronchiectasis and cysts are probably underrated complications of COVID-19

- There are no cases in the literature that clarifies temporal and radiological evolution of atypical findings such as pneumothorax and cysts in COVID-19 pneumonia

- Pneumothorax, pneumomediastinum and cysts as complications of COVID-19 can worsen prognosis, in terms of prolonged hospitalization and need of oxygen therapy.

\section{Correspondence: Giorgio Emanuele Polistina, Sub-Intensive Care Unit and Respiratory Physiopathology Department, Cotugno-Monaldi Hospital, Via L. Bianchi 1, Naples, 80121, Italy. E-mail: giorgiopolistina@gmail.com}

Key words: COVID-19; Coronavirus; pneumonia; pneumomediastinum; pneumothorax; high resolution computed tomography.

Acknowledgments: We would like to thank all our colleagues of Cotugno Hospital in Naples, who are fighting every day with us this invisible enemy.

Contributions: GEP, FS, conception and design of the study, manuscript drafting; PI, ML, data acquisition and analysis; GF, AA, methodology; GF, project administration. All the authors have read and approved the final version of the manuscript and agreed to be accountable for all aspects of the work.

Conflict of interest: The authors declare no conflict of interest.

Registration of research studies: Not applicable.

Guarantors: Giorgio Emanuele Polistina, MD.

Ethics approval and consent to participate: No ethical committee approval was required for this case series by the Division of Respiratory Physiopathology, Monaldi Hospital, Naples, Italy.

Consent for publication: Written informed consent was obtained from the patient for publication of this case report and accompanying images. A copy of the written consent is available for review by Editor-in-Chief of this journal on request.

Funding: None.

Received for publication: 1 July 2020.

Accepted for publication: 24 July 2020.

${ }^{\circ}$ Copyright: the Author(s), 2020

Licensee PAGEPress, Italy

Monaldi Archives for Chest Disease 2020; 90:1482

doi: 10.4081/monaldi.2020.1482

This article is distributed under the terms of the Creative Commons Attribution Noncommercial License (by-nc 4.0) which permits any noncommercial use, distribution, and reproduction in any medium, provided the original author(s) and source are credited.

\begin{abstract}
The coronavirus disease 2019 (COVID-19) is a recent pandemic that affected more than 5 million people worldwide. Chest high resolution computed tomography (HRCT) is an essential tool in diagnosis and management of the disease. Pulmonary parenchymal opacity is a typical sign of the disease, but not the only one. Pneumothorax, pneumomediastinum, bronchiectasis and cysts are probably underrated complications of COVID-19 that can worsen prognosis, in terms of prolonged hospitalization and need of oxygen therapy. In our single center case series, we outline four different manifestations of pneumothorax, pneumomediastinum and cysts in hospitalized patients with COVID-19 pneumonia.
\end{abstract}

\section{Introduction}

Since December 2019, an increasing number of pneumonia cases caused by the severe acute respiratory syndrome coronavirus 2 (SARS-CoV-2) emerged in Wuhan, Hubei, China, and subsequently, a pandemic of coronavirus disease 2019 (COVID19) swept the globe $[1,2]$.

The novel coronavirus was identified on January 6, 2020, and was speculated to be linked to the Huanan Seafood Market in Wuhan. As of May 25, 2020, more than 5204508 cases have been confirmed worldwide, with 337687 related deaths [3]. Furthermore, the virus is still spreading at an alarming rate. A wide variety of CT findings in COVID-19 were reported in several studies but pneumothorax and pneumomediastinum were described as rare and less common. It was also observed that pleural effusion, pericardial effusion, lymphadenopathy, cavitation, CT halo sign and pneumothorax are uncommon but possibly seen with disease progression [4]. To date, there is lack of data in literature of pneumothorax and pneumomediastinum as onset finding or early complication of COVID-19 pneumonia. 
This case series aims to outline radiological manifestations of pneumothorax, pneumomediastinum and cysts in different stages of COVID-19 pneumonia. We retrospectively analyzed patients admitted in our Department of Sub-Intensive Care Unit of Cotugno Hospital in Naples, Italy.

\section{Case \#1}

A 44-years-old man, smoker, was admitted in our Department after onset at home of sudden chest pain, high fever $\left(>40^{\circ}\right)$ and severe shortness of breath. Screening tests were negative for multiple respiratory pathogens, infection from SARS-CoV-2 was confirmed on swab test in reverse transcription polymerase chain reaction (RT-PCR) assay. Blood gas analysis (ABG) showed on fraction of inspired $\mathrm{O} 2\left(\mathrm{FiO}_{2}\right)$ at $21 \%$ pO2 $57 \mathrm{mmHg} ; \mathrm{PCo}_{2} 31$ mmHg; $\mathrm{Ph} 7.47$, Lac 1,6 mmol/L with $\mathrm{PaO}_{2} / \mathrm{FiO}_{2}$ ratio (P/F) 319 and therefore treated with oxygen therapy with nasal cannula with gas flow rate of $21 / \mathrm{min}$.

HRCT scan showed multiple areas of ground glass opacities (GGO) on upper right lobe and upper left lobe. On left basal lobe evidence of spontaneous pneumothorax and ipsilateral extensive subcutaneous emphysema (Figure 1a). Patient was treated with empiric antibiotics, hydroxychloroquine and pleural drainage. Follow up at 14 days showed complete resolution of pneumothorax and subcutaneous emphysema with residual pleural and parenchymal thickening (Figure 1b).

\section{Case \#2}

A 58-years-old male, former smoker, with no relevant medical history, attended emergency room in March 2020. He referred onset of fever $\left(39.5^{\circ} \mathrm{C}\right)$, sore throat, dry cough, and mild shortness of breath 7 days before (day 1). At admission he also showed a mild acute respiratory distress syndrome (ARDS), P/F being $270 \mathrm{mmHg}$. SARS-CoV-2 was confirmed by RT-PCR assay on nasopharyngeal swab. A HRCT revealed multiple patchy GGO and sporadic bronchiectasis (Figure 2a). We administered oxygen therapy via Venturi mask at $\mathrm{FiO}_{2} 28 \%$ to reach a SpO2 $>94 \%$ and start medical therapy with hydroxychloroquine, azithromycin, antivirals, low molecular weight heparin (LMWH). IL-6 was elevated, therefore Tocilizumab was added. Within days the patient recovered from fever and cough, but he still needed oxygen and IL-6 was

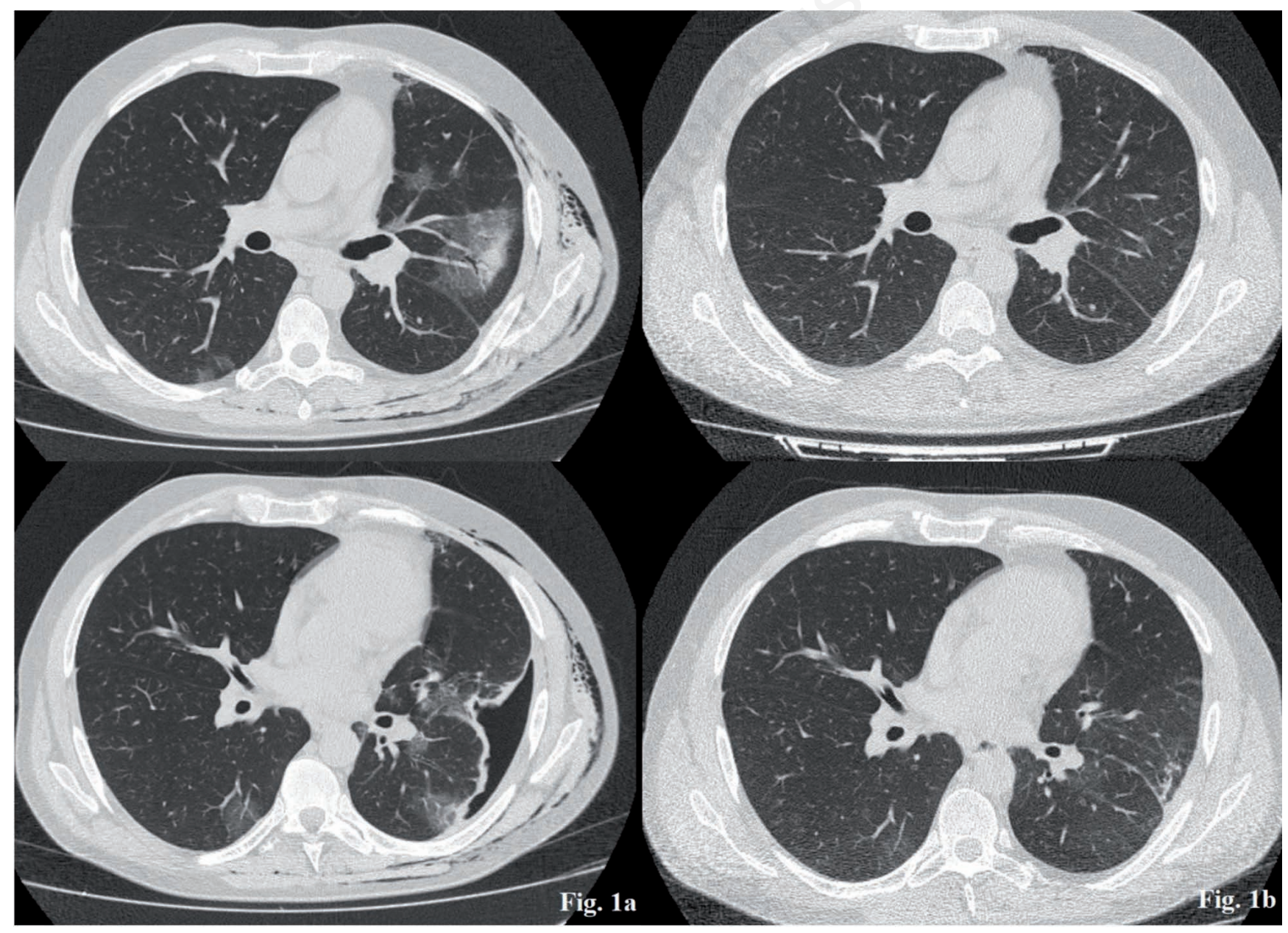

Figure 1. a) CT localization of ground glass opacities (GGO) on upper left lobe and left basal lobe evidence of spontaneous pneumothorax and ipsilateral extensive subcutaneous emphysema. b) Complete resolution of pneumothorax and subcutaneous emphysema with residual pleural and parenchymal thickening. 
persistently elevated despite anti IL-6 receptor treatment. A follow up HRCT was performed (day 17), showing persistent and extensive GGO; a thick-walled cyst with maximum diameter of $25 \mathrm{~mm}$ was observed distally from an evident bronchiectasis (Figure 2b).

\section{Case \#3}

On March $7^{\text {th }} 2020$ (day 1), a 57-years-old man was admitted to ICU due to fever, dry cough and severe ARDS with $\mathrm{P} / \mathrm{F}<100$. The patient had neither smoking nor respiratory medical history, but he reported systemic hypertension. RT-PCR on nasopharyngeal swab detected SARS-CoV-2. HRCT scan showed bilateral GGO, involving all lobes. Bibasal consolidations and traction bronchiectasis were also detected (Figure 3a). The laboratory testing showed a peculiar inflammatory pattern characterized by elevated serum C-reactive protein (CRP) and ferritin, leukopenia, as well as elevated interleukin-2 receptor (IL2-R) and interleukin-6 (IL6). The patient underwent low-volume low-pressure mechanical ventilation for 21 days; he also received hydroxychloroquine, antivirals, LMWH, and Tocilizumab. On April $4^{\text {th }}$ (day 29), a second HRCT showed a remarkable regression of GGO, bibasal consolidations prevailed instead. Moreover, fibrotic strands and architectural distortion were predominant in non-dependent areas of the lungs, where cystic airway enlargement replaced a cylindrical bronchiectasis (Figure 3b). Despite clinical and radiological improvement, systemic inflammation persisted as assessed by CRP, ferritin and IL-6. Noticeably a follow up HRCT on May $2^{\text {nd }}$ (day 57), detected a substantial regression of all anomalies.

\section{Case \#4}

A 58-years-old male presented to the emergency room with "flulike symptoms" and shortness of breath. He was a nurse with ascertained Sars-CoV-2 workplace exposure. No smoking history

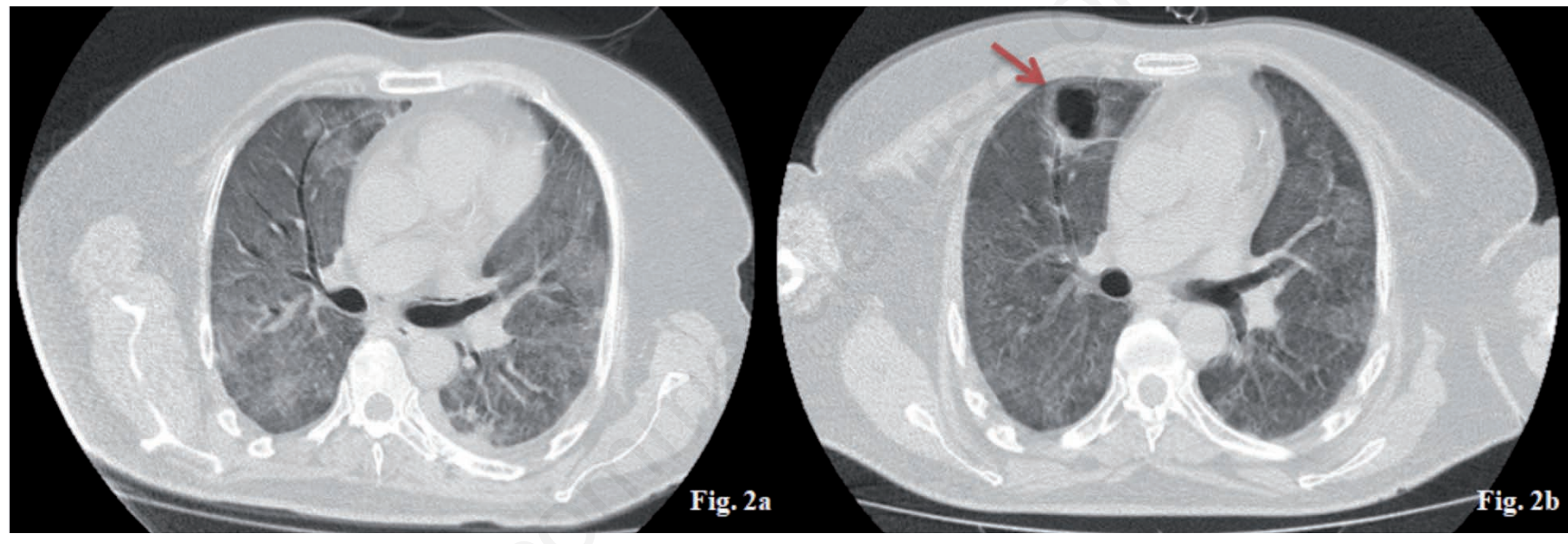

Figure 2. a) Diffuse GGO and sporadic bronchiectasis. b) $25 \mathrm{~mm}$ diameter, thick walled cyst distally from a bronchiectasis (arrow).

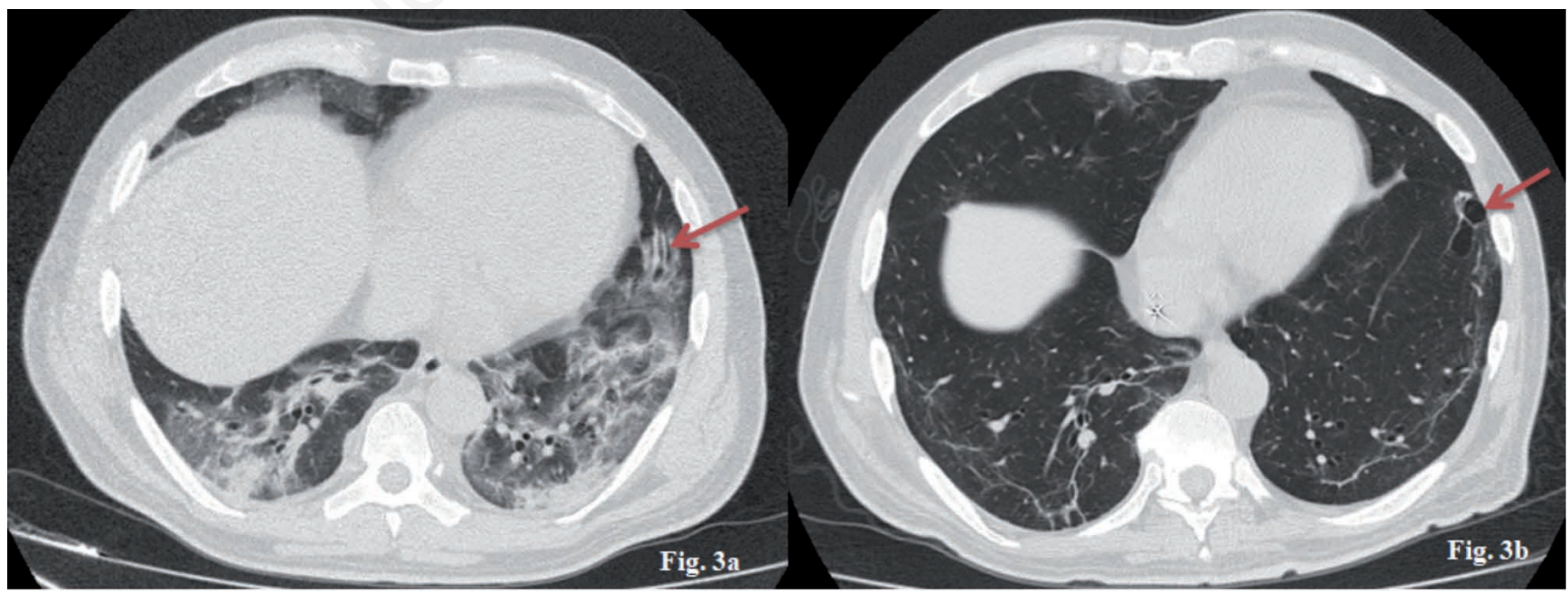

Figure 3. a) GGO, bibasal consolidations and traction bronchiectasis (arrow). b) Cystic airway enlargement replaced a cylindrical bronchiectasis (arrow). 
and no comorbidities were reported. $\mathrm{ABG}$ with $\mathrm{FiO}_{2}$ at $21 \%$ showed $\mathrm{pO}_{2} 68 \mathrm{mmHg} ; \mathrm{pCo}_{2} 31 \mathrm{mmHg}$; $\mathrm{pH} 7.48$ with $\mathrm{P} / \mathrm{F} 323$, no oxygen therapy was immediately started. HRTC scan showed bilateral, peripheral GGOs involving all lobes. Partial consolidations with septal thickening were observed on left basal lobe (Figure 4a). On third day $\mathrm{ABG}$ declined so we started oxygen therapy with high flow nasal cannula with Fio2 70\% and flow 50 1/min to reach $\mathrm{SpO}_{2}$ $>93 \%$. On fifth day from admission, his symptoms and pulmonary opacities worsened and hence he was transferred to ICU and mechanically ventilated. Medications during hospitalization included hydroxychloroquine, azithromycin, and other supportive drug treatments. Patient developed acute renal failure and a leftsided tension pneumothorax while in ICU (Figure 4b), treated with chest tube insertion. Despite that, his clinical condition deteriorated, a right heart failure occurred, and the patient died on day 10.

\section{Discussion}

COVID-19 is an acute respiratory infection caused by SARSCoV 2. Patients with COVID-19 mainly manifest with fever, cough and dyspnea. Severe patients usually develop dyspnea and/or hypoxemia after 1 week of onset, and progress rapidly to ARDS and coagulation dysfunction [5]

Due to its high sensitivity and ease-of-use, HRTC is an important screening tool for suspected COVID-19 patients. The most common patterns and distribution of CT findings in COVID19 pneumonia are ground glass opacities $(88.0 \%)$, bilateral involvement $(87.5 \%)$, peripheral distribution $(76.0 \%)$, and multi lobar (more than one lobe) involvement (78.8\%) [4]. Other less common CT findings include interlobular septal thickening, bronchiectasis, pleural thickening, and subpleural involvement $[6,7]$. Pleural effusion, pericardial effusion lymphadenopathy, cavitation, CT halo sign, and pneumothorax are rare [8]. Isolated GGO or a combination of GGO and consolidations are some of the most common CT findings on hospital admission of COVID-19 pneumonia due to probable alveolar swelling and septal inflammation caused by infection [9].

We could not find in the literature any case that clarifies temporal and radiological evolution of atypical findings such as pneumothorax and cysts in COVID-19 pneumonia. Sporadic cases of spontaneous pneumothorax are reported in the literature to support the exceptional early complication [10,11]. One case reported these findings developed during course of infection [12].

The cases we reported, occurred during Italian COVID-19 pneumonia outbreak, demonstrated a different radiological evolution of the disease. Pneumothorax, pneumomediastinum and cysts are probably underrated complications of COVID-19 that can worsen prognosis, in terms of prolonged hospitalization and need of oxygen therapy. Mediastinal emphysema and pneumothorax are well-known complications of mechanical ventilation or complications from previous alterations. It has also been reported that late stage ARDS is characterized by fibrosis and interstitial

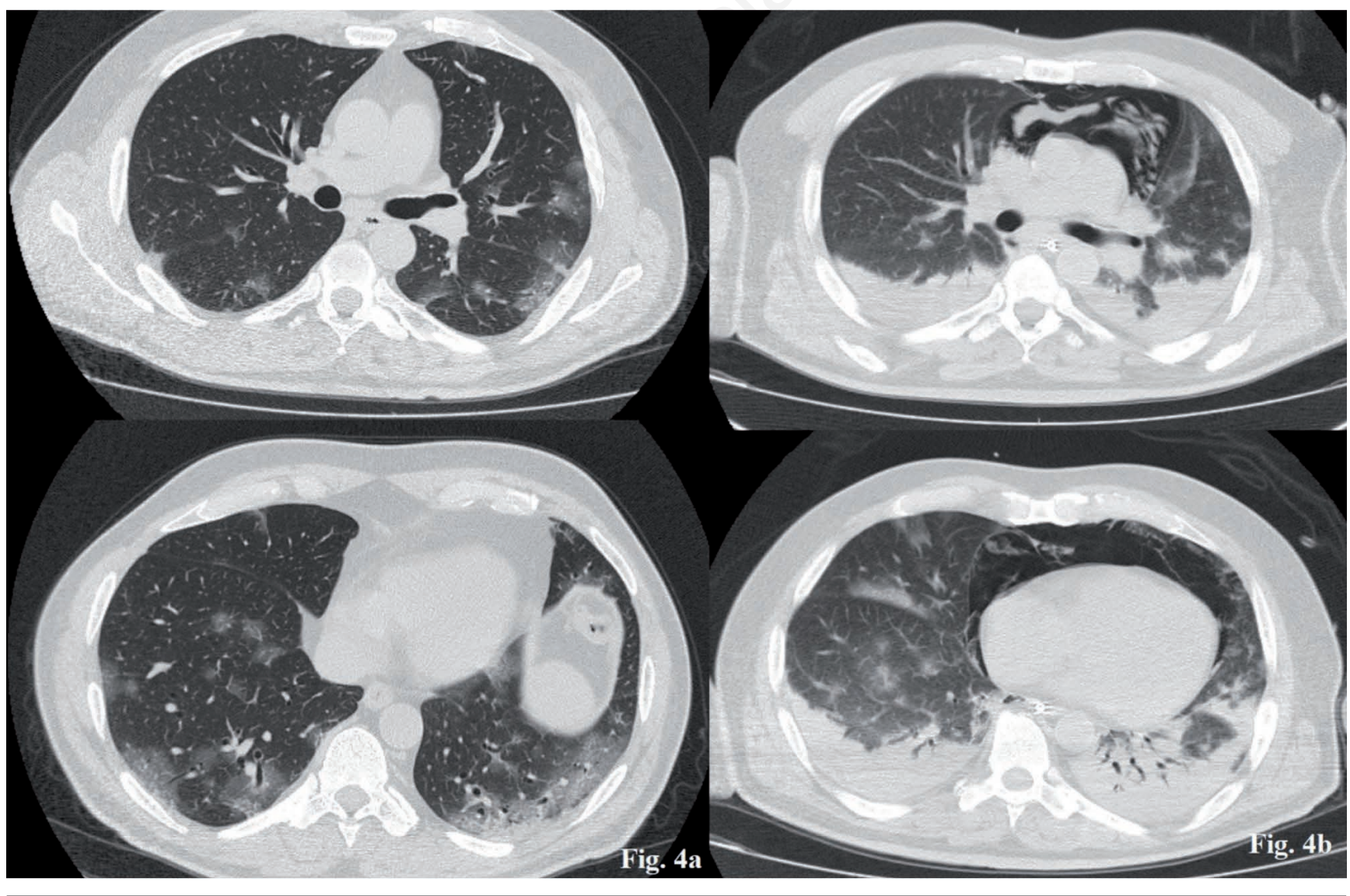

Figure 4. a) Bilateral multiple ground glass areas, predominantly subpleural, tending to confluence and to thickening in left basal lobe. b) Marked worsening of ground glass areas. Bibasal consolidations with traction bronchiectasis and left-sided tension pneumothorax. 
distortion that can lead to subpleural cysts or bullae usually in ventilated patients [13]. This case series highlights that such complications can exist in non-ventilated patient as well. Moreover, the patients we reported did not have a history of pneumothorax or any preexisting pulmonary disease.

A hypothesis is that alveoli may be prone to rupturing due the diffuse alveolar injury in COVID-19 pneumonia; pronounced cough may have caused alveolar rupture. Mediastinal emphysema results from a sudden increase in the alveolar pressure, causing alveolar rupture and air leakage with interstitial emphysema [14].

It has been recently demonstrated by lung biopsies, that COVID-19 ARDS involves cellular fibromixoid exudates, hyaline membrane formation and inflammatory infiltrates [15]. Different hypothesis could be based on pulmonary fibrosis caused by inflammation, bronchioles are narrow and distorted and the valve mechanism could case pulmonary bullae. Increased intrapulmonary pressure, such as coughing and mechanical ventilation, may lead to bullae rupture and secondary pneumothorax [11]. Nevertheless, atypical CT findings occurred during both mechanical ventilation and standard oxygen therapy, independently from baseline symptoms. This observation suggests that underlying physiopathological alterations could predispose COVID-19 patients to those complications.

In conclusion, on this COVID-19 pneumonia outbreak, clinicians and radiologists should be alerted to the possibility to observe pneumothorax and pneumomediastinum, as early manifestation or treatment complication, more frequently than reported on literature.

\section{References}

1. World Health Organization. Readiness is the key to detect, combat spread of the new coronavirus. Accessed: 27 Jan 2020. Available from: https://www.who.int/southeastasia/news/detail/ 27-01-2020-readiness-is-the-key-to-detect-combat-spread-of-thenew-coronavirus

2. World Health Organization. WHO confirms first cases of novel coronavirus (2019-nCoV) in the Eastern Mediterranean Region. Accessed: 29 Jan 2020. Available from: http://www.emro.who. int/media/news/who-confirms-first-cases-of-novel-coronavirus2019-ncov-in-the-eastern-mediterranean-region.html

3. World Health Organization. Coronavirus disease 2019 (COVID19) situation report-125. Available from: https://www.who. int/docs/default-source/coronaviruse/situationreports/20200524-covid-19-sitrep-125.pdf?sfvrsn=80e7d7f0_2 4. Salehi S, Abedi A, Balakrishnan S, Gholamrezanezhad A. Coronavirus disease 2019 (COVID-19): A systematic review of imaging findings in 919 patients. Am J Roentgenol 2020: 215:87-93

5. Wang D, Hu B, Hu C, et al. Clinical characteristics of 138 hospitalized patients with 2019 novel coronavirus-infected pneumonia in Wuhan, China. JAMA 2020:e201585.

6. Lei J, Li J, Li X, Qi X. CT imaging of the 2019 novel coronavirus (2019-nCoV) pneumonia. Radiology 2020;295:18.

7. Shi H, Han X, Zheng C. Evolution of CT manifestations in a patient recovered from 2019 novel coronavirus (2019-nCoV) pneumonia in Wuhan, China. Radiology 2020;295:20.

8. Kong W, Agarwal PP. Chest imaging appearance of COVID-19 infection. Radiol Cardiothorac Imaging 2020;2:e200028.

9. Xu Z, Shi L, Wang Y, et al. Pathological findings of COVID-19 associated with acute respiratory distress syndrome. Lancet Respir Med 2020;8:420-2.

10. Lyu R, Li X. Diagnosis and treatment of severe COVID-19 complicated with spontaneous pneumothorax: a case report. Adv Ultrasound Diagn Ther 2020;02:142-6.

11. Aydin S, Öz G, Dumanlı A, Balcı A, Gencer A. A case of spontaneous pneumothorax in Covid-19 pneumonia. J Surg Res 2020;3:96-101.

12. Sun R, Liu H, Wang X. Mediastinal emphysema, giant bulla, and pneumothorax developed during the course of covid-19 pneumonia. Korean J Radiol 2020;21:541-4.

13. Gattinoni L, Caironi P, Pelosi P, Goodman LR. What has computed tomography taught us about the acute respiratory distress syndrome? Am J Respir Crit Care Med 2001;164:1701-11.

14. Park SJ, Park JY, Jung J, Park SY. Clinical manifestations of spontaneous pneumomediastinum. Korean J Thorac Cardiovasc Surg 2016;49:287-91.

15. Xu Z, Shi L, Wang Y, et al. Pathological findings of COVID-19 associated with acute respiratory distress syndrome. Lancet Respir Med 2020;8:420-2. 